\title{
Nephrotoxicity and Kidney Transport Assessment on 3D Perfused Proximal Tubules
}

\author{
Marianne K. Vormann, ${ }^{1}$ Linda Gijzen, ${ }^{1}$ Simon Hutter, ${ }^{1}$ Lisette Boot, ${ }^{1}$ Arnaud Nicolas, ${ }^{1}$ \\ Angelique van den Heuvel, ${ }^{1}$ Jelle Vriend, ${ }^{2}$ Chee Ping $\mathrm{Ng}^{1},{ }^{1}$ Tom T. G. Nieskens, ${ }^{2}$ Vincent van Duinen, ${ }^{3}$ \\ Bjorn de Wagenaar, ${ }^{1}$ Rosalinde Masereeuw, ${ }^{4}$ Laura Suter-Dick, ${ }^{5}$ Sebastiaan J. Trietsch, ${ }^{1}$ Martijn Wilmer, ${ }^{2}$ \\ Jos Joore, ${ }^{1}$ Paul Vulto, ${ }^{1}$ and Henriette L. Lanz ${ }^{1,6}$
}

Received 3 April 2018; accepted 23 July 2018; published online 14 August 2018

\begin{abstract}
Proximal tubules in the kidney play a crucial role in reabsorbing and eliminating substrates from the body into the urine, leading to high local concentrations of xenobiotics. This makes the proximal tubule a major target for drug toxicity that needs to be evaluated during the drug development process. Here, we describe an advanced in vitro model consisting of fully polarized renal proximal tubular epithelial cells cultured in a microfluidic system. Up to 40 leak-tight tubules were cultured on this platform that provides access to the basolateral as well as the apical side of the epithelial cells. Exposure to the nephrotoxicant cisplatin caused a dose-dependent disruption of the epithelial barrier, a decrease in viability, an increase in effluent LDH activity, and changes in expression of tight-junction marker zonaoccludence 1, actin, and DNA-damage marker H2A.X, as detected by immunostaining. Activity and inhibition of the efflux pumps P-glycoprotein (P-gp) and multidrug resistance protein (MRP) were demonstrated using fluorescence-based transporter assays. In addition, the transepithelial transport function from the basolateral to the apical side of the proximal tubule was studied. The apparent permeability of the fluorescent P-gp substrate rhodamine 123 was decreased by $35 \%$ by co-incubation with cyclosporin A. Furthermore, the activity of the glucose transporter SGLT2 was demonstrated using the fluorescent glucose analog 6NBDG which was sensitive to inhibition by phlorizin. Our results demonstrate that we developed a functional 3D perfused proximal tubule model with advanced renal epithelial characteristics that can be used for drug screening studies.
\end{abstract}

KEY WORDS: kidney-on-a-chip; nephrotoxicity; apparent permeability $\left(P_{\text {app }}\right)$; proximal tubule; transepithelial transport.

Guest Editors: Rosalinde Masereeuw and Raymond Lai

Electronic supplementary material The online version of this article (https://doi.org/10.1208/s12248-018-0248-z) contains supplementary material, which is available to authorized users.

${ }^{1}$ MIMETAS B.V, JH Oortweg 19, $2333 \mathrm{CH}$, Leiden, the Netherlands.

${ }^{2}$ Department of Pharmacology and Toxicology, Radboud UMC, P.O. box 9101, $6500 \mathrm{HB}$, Nijmegen, the Netherlands.

${ }^{3}$ Division of Analytical Biosciences, Leiden Academic Center for Drug Research, Einsteinweg 55, 2333CC, Leiden, the Netherlands.

${ }^{4}$ Division Pharmacology, Department of Pharmaceutical Sciences, Utrecht University, Universiteitsweg 99, 3584 CG, Utrecht, the Netherlands.

${ }^{5}$ School of Life Sciences, University of Applied Sciences Northwestern Switzerland, Gründenstrasse 40, 4132, Muttenz, Switzerland.

${ }^{6}$ To whom correspondence should be addressed. (e-mail: h.lanz@mimetas.com)

\section{INTRODUCTION}

Renal proximal tubules play a crucial role in reabsorbing salt, water, and organic solutes such as glucose from the glomerular filtrate as well as eliminating endogenous and exogenous waste products from the body (1). The transepithelial transport of substrates and the concentration of xenobiotics in the tubular lumen make the proximal tubule a target for drug-induced toxicity (2).

Currently, preclinical assessment of nephrotoxicity is mainly performed in animal studies. However, due to ethical concerns and the limited translatability of these models to the human situation, in vitro modeling is rapidly becoming important for studying solute transport, drug-induced toxicity, and disease-related kidney failure (3). Current-day in vitro models typically comprise human renal proximal tubule epithelial cells (RPTECs) grown on a permeable membrane 
support. But, these systems often lack elements such as flow or embedding in an extracellular matrix (ECM) structure and are not compatible with image-based readouts. Moreover, the permeable support membrane is a crucial barrier influencing both transport parameters and physiology of cells.

In recent years, the use of microfluidics has gained significant interest for building human tissue models of enhanced physiological relevance. These techniques, popularly referred to as organ-on-a-chip, add flow to cell culture systems, enable gradient formation, facilitate a 3D architecture of tissues, allow engineering of tissue complexity through layered co-cultures, and are typically compatible with ECMembedded cultures. Various model systems for the renal proximal tubule have been reported over the past few years (4-7). Although they represent powerful examples of the added value of microfluidics to the realm of in vitro kidney modeling, these chips are largely prototypes, yield single data points per chip, require external tube and pump connections for each chip, and are typically constructed of materials that absorb hydrophobic compounds (8).

The current challenge is to implement these prototypes into platforms combined with protocols and assays for routine use in an end-user environment $(9,10)$. Robust cell culture protocols are needed that can be performed in parallel in order to test dilution series, including several replicates and appropriate controls. Furthermore, the platform needs to be compatible with a range of assays that are typically used in an in vitro environment, including fluorescence-based methods, immunohistochemical staining, barrier integrity monitoring, transport studies, viability assays, qRT-PCR, ELISAs, and many others. Last but not the least, operation of the platform should be straightforward to the level that expert microfluidic skills are not required for end-users. Therefore, the organ-ona-chip platform needs to be compatible with standard lab equipment such as pipets, (confocal) microscopes, plate readers, and other microwell-plate compatible equipment.

In this research, we used the OrganoPlate (11), a microtiter-plate-based microfluidic chip platform enabling 40 tissues per plate. A proximal tubule-on-a-chip was modeled with renal proximal tubule epithelial cells (12) (RPTEC; SA7K clone) grown as perfused tubules against an ECM.

The cells used for this study were developed and characterized by Li et al. (12) and showed similar expression levels of several uptake and efflux transporters when compared to human primary proximal tubule cells. The RPTEC line further showed improved uptake and efflux compared to the HK-2 cell line, with a more sensitive detection of nephrotoxicants. Two of the efflux transporters, P-glycoprotein (P-gp) and multidrug resistance-associated protein 4 (MRP4), showed high levels of expression. Furthermore, the morphology of cell monolayers showed a typical cobblestone structure which is important for tight barriers.

After optimization of growth conditions of RPTEC in the OrganoPlate, tubules were analyzed for polarization of the epithelial layer by immunostaining and barrier integrity through a live fluorescent dye assay. Next, the platform was evaluated for its suitability in studying (transepithelial) transport and drug-induced toxicity. The technology can be implemented in every basic cell laboratory with standard laboratory equipment and can be assessed with multiplexed readouts.

\section{MATERIALS AND METHODS}

\section{Cell Culture}

Renal proximal tubule epithelial cells (RPTEC; Kidney PTEC Control Cells, SA7K Clone, Sigma, Germany, MTOX1030) were cultured on PureCol-coated (Advanced BioMetrix, 5005-B, diluted with 1:30 in HBSS (Sigma H6648), 20 min incubation at $37^{\circ} \mathrm{C}$ ) $\mathrm{T} 75$ flasks in MEME alpha Modification (Sigma, M4526) supplemented with RPTEC Complete Supplement (Sigma, MTOXRCSUP), L-glutamine (1.87 mM, Sigma, G7513), gentamicin (28 $\mu \mathrm{g} / \mathrm{mL}$, Sigma, G1397), and amphotericin B (14 ng/mL, Sigma, A2942). Cells were incubated in a humidified incubator $\left(37^{\circ}, 5 \% \mathrm{CO}_{2}\right)$, and every 2-3 days, the medium was changed. At $90-100 \%$ confluency, cells were washed with HBSS (Sigma, H6648), detached with accutase (Sigma, A6964), pelleted (140 g, $5 \mathrm{~min}$ ), and used for seeding in the OrganoPlate. Cells for experiments were used up to passage 3 .

\section{OrganoPlate Culture}

For all experiments, a three-lane OrganoPlate (Mimetas $\mathrm{BV}, 4003$ 400B) with a channel width of $400 \mu \mathrm{m}$ and a height of $220 \mu \mathrm{m}$ was used. $1.6 \mu \mathrm{L}$ of extracellular matrix (ECM) gel composed of $4 \mathrm{mg} / \mathrm{mL}$ collagen 1 (AMSbio Cultrex 3D Collagen I Rat Tail, Cat. 3447-319 020-01), 100 mM HEPES (Life Technologies, 15630), and $3.7 \mathrm{mg} / \mathrm{mL} \mathrm{NaHCO}$ (Sigma, 320 S5761) was injected into the middle inlet (Fig. 1a) of all 40 chips. After a polymerization time of $20 \mathrm{~min}, 20 \mu \mathrm{L} \mathrm{HBSS}$ was added on top of the collagen 1 and the plate was incubated in a humified incubator at $37^{\circ} \mathrm{C}$ over night. After polymerization of the ECM, the plate could be also stored in a humified incubator $\left(37^{\circ} \mathrm{C}\right)$ for up to a week. RPTEC were detached and resuspended in medium at a concentration of $10 \times 10^{6}$ cells per $\mathrm{mL}$. Two microliters of the cell suspension $\left(20 \times 10^{3}\right.$ cells $)$ was injected into each top inlet, followed by an addition of $50 \mu \mathrm{L}$ medium to the same well. For control chips, $2 \mu \mathrm{L}$ of medium was injected into the top inlet instead of the cell suspension. Subsequently, the OrganoPlate was placed for $5 \mathrm{~h}$ at an angle of $70^{\circ}$ in the incubator $\left(37^{\circ} \mathrm{C}, 5 \% \mathrm{CO}_{2}\right.$, humidified). After attachment of the cells, $50 \mu \mathrm{L}$ of medium was added to the top outlet, bottom inlet, bottom outlet (Fig. 1a), and HBSS on the gel was removed. The OrganoPlate was placed flat in an incubator on an interval rocker platform $\left( \pm 7^{\circ}\right.$ angle, $8 \mathrm{~min}$ interval) enabling a bidirectional flow though the perfusion channels (see Fig. 5S). At day 3, antibiotics (gentamycin and amphotericin B) were removed from the medium. The medium was replaced every 2-3 days. Forty-eight-hour toxicant exposures were started at day 6; all other experiments were performed at days $7,8,9$, or 10 . To show the effect of flow in the system, an OrganoPlate was taken off the rocker platform from day 1 to day 4. At day 4, the medium was refreshed and the plate was rocked again under same conditions as the control experiments.

\section{Immunohistochemistry}

RPTEC tubes were fixed by replacing the medium with $3.7 \%$ formaldehyde (Sigma, 252549) in HBSS (Sigma, 

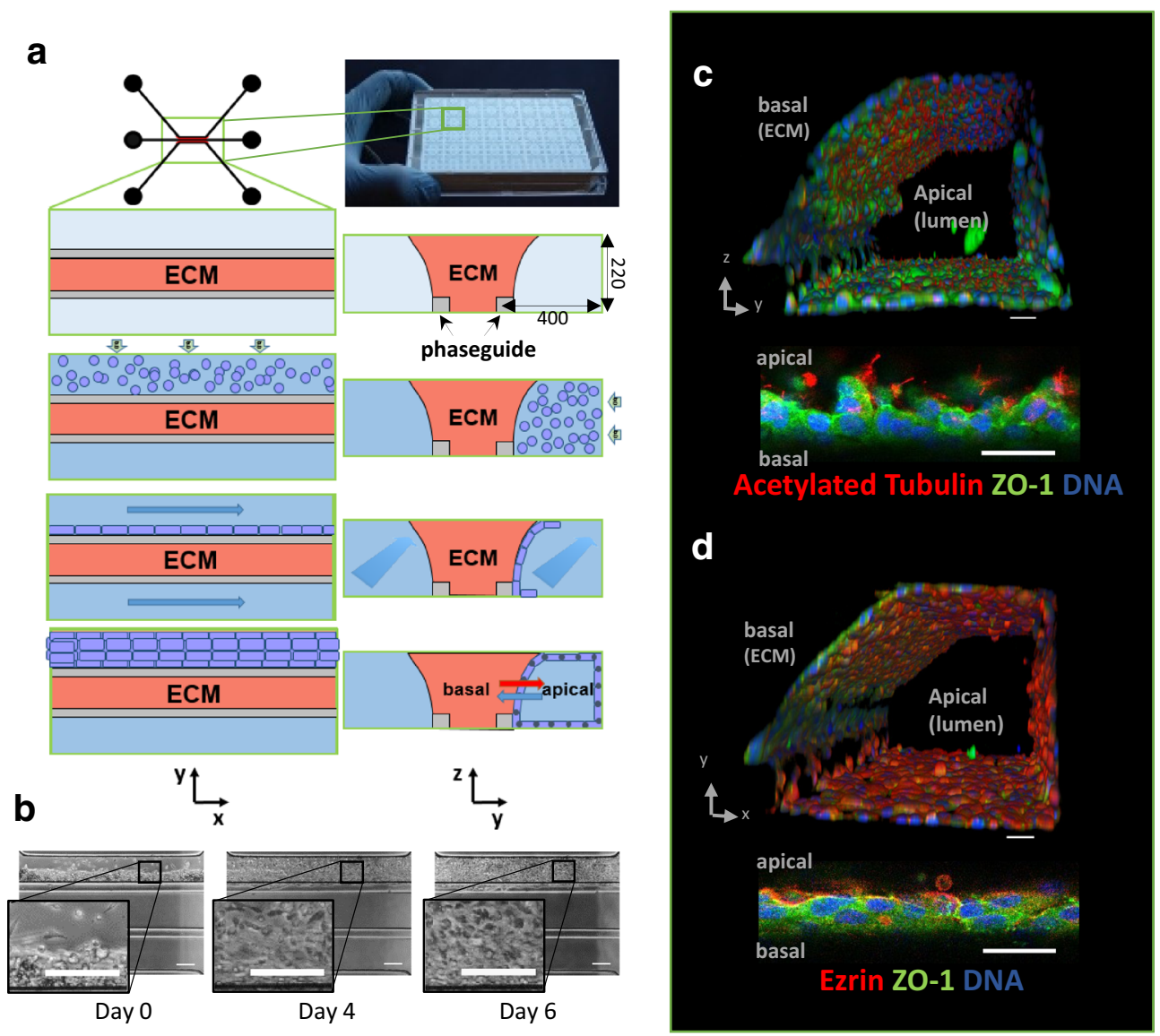

Fig. 1. Proximal tubule-on-a-chip model in the three-lane OrganoPlate platform. a Seeding RPTEC against collagen 1: After loading collagen 1 into the middle channel, cells were seeded in the adjacent channel. By gravity, cells are triggered to attach to the gel. After inducing flow, RPTEC start growing a perfused tubular structure. Dimensions of the channels are in micrometer. b Phase contrast images at day 0, day 4, and day 6 after seeding of RPTEC. Images show that RPTEC form a tubular structure in the top channel in 6 days. Scale bar $=200 \mu \mathrm{m}$. c, $\mathbf{d}$ 3D reconstruction images of RPTEC tubules in the OrganoPlate showing a view into the lumen of tubules (apical side). The magnification shows a single z-slice of the cells growing against the ECM. Nuclei in blue. Scale bars $=30 \mu \mathrm{m}$. $\mathbf{c}$ Image of the tubule showing the presence of cilia (acetylated tubulin, red) pointing into the direction of the lumen. The confluent tubules express ZO-1 at the cell borders which confirms the tight junction formation between neighboring epithelial (green). d Ezrin expression (red) on the apical side and ZO-1 (green) expression at the cell borders of the tubules shows the correct polarization of the cells

55037C) for $10 \mathrm{~min}$. Tubules were washed with washing solution (4\% fetal bovine serum (Gibco, 16140-071) in HBSS) and permeabilized $(0.3 \%$ Triton X-100 (Sigma, T8787) in HBSS for $10 \mathrm{~min}$. Next, cells were incubated for $45 \mathrm{~min}$ in blocking solution (2\% FBS, $2 \%$ bovine serum albumin (BSA) (Sigma, A2153), and 0.1\% Tween 20 (Sigma, P9416) in HBSS). Hereafter, cells were incubated with the primary antibodies, diluted in blocking solution, for $60 \mathrm{~min}$ at room temperature. Primary antibodies against Ms-a-ezrin (BD Biosciences, 610602, 1:200), Ms-a-acetylated tubulin (Sigma, T6793, 1:4000), Rb-a-Zonula occludens-1 (ZO-1) (Thermo Fischer, 61-7300, 1:125, rabbit), Rb-a-Phospho-Histone (H2A.X) (Cell Signaling Technology, 9718S, 1:200, rabbit), mouse isotype (Life technologies, 86599), and rabbit isotype (Life technologies, 86199) were used. Subsequently, cells were washed three times with washing solution and then incubated for $30 \mathrm{~min}$ at room temperature with secondary antibodies Gt-a-Ms IgG $(\mathrm{H}+\mathrm{L})$ Alexa Fluor 555 (Life Technologies, A21422, 1:250), Gt-a-Rb IgG (H+L) Alexa Fluor 488 (Life Technologies, A32731, 1:250) diluted in blocking solution. After washing the tubules three times, nuclei were stained with DraQ5 (Abcam, ab108410, 1:1000) or Nucblue-fixed cell stain (Life Technologies, R37606, 2 drops/mL) or Actin red (Life Technologies, R37112, 2 drops/ $\mathrm{mL}$ ) in the last washing step. Fluorescent images for the 3D reconstructions were taken with the Leica SP5-Sted Confocal Microscope. A z-stack of $220 \mu \mathrm{m}$ with $2 \mu \mathrm{m}$ between each image plane was imaged with Alexa 488, Alexa 555, and Alexa 647. Fluorescent images for the analysis of the protein expression after a toxicant exposure were taken with the ImageXpress ${ }^{\circledR}$ Micro Confocal High-Content Imaging System (Molecular Devices). A z-stack of $5 \mu \mathrm{m}$ between each image plane was imaged for DAPI, FITC, TRITC, and Cy5 channels. A maximum projection was created for depicting 
the images, and a summary projection was used for quantifying the fluorescent intensity of the markers.

\section{Barrier Integrity Assay}

The barrier integrity assay (BI assay) was performed by replacing the medium of the perfusion channel with medium containing $0.5 \mathrm{mg} / \mathrm{mL}$ TRITC-dextran (4.4 kDa, Sigma, FD20S) and $0.5 \mathrm{mg} / \mathrm{mL}$ FITC-dextran (155 kDa, Sigma, T1287). Next, the plate was imaged every $2 \mathrm{~min}$ for $12 \mathrm{~min}$ with the ImageXpress Micro XLS-C High Content Imaging System (Molecular Devices) at $37^{\circ} \mathrm{C}$. Leakage of the dyes from the apical side of the tube to the basal side into the ECM was measured, and the ratio between the basal and the apical was analyzed with Fiji (13). The labeled dextrans can be washed out after each measurement. The permeability of the membranes was analyzed by measuring the amount of molecules which leaked though the membrane into the adjacent gel lane over time. From these measurements, the apparent permeability index $\left(P_{\text {app }}\right.$ : initial flux of a compound through a membrane, normalized by membrane surface area and donor concentration) was calculated by the following formula:

$P_{\text {app }}=\frac{\Delta C_{\text {receiver }} \times V_{\text {receiver }}}{\Delta \mathrm{t} \times A_{\text {barrier }} \times C_{\text {donor }}}\left(\frac{\mathrm{cm}}{\mathrm{s}}\right)$

$\Delta C_{\text {receiver }}$ is the measured normalized intensity difference of the ECM to the donor channel (Fig. $2 b$ value of D/value of C) at $t_{0 \mathrm{~min}}$ and $t_{10 \mathrm{~min}}, V_{\text {receiver }}$ is the volume of the measured region in the ECM channel (Fig. 2b, c; channel height $\times$ channel width $\times$ channel length $=220 \mu \mathrm{m} \times 2304 \mu \mathrm{m} \times$ $\left.204.8 \mu \mathrm{m}=0.0001 \mathrm{~cm}^{2}\right), \Delta t$ is the time difference $t_{10} \min -$ $t_{0 \mathrm{~min}}=10 \mathrm{~min}, A_{\text {barrier }}\left(0.0057 \mathrm{~cm}^{2}\right)$ is the surface of the ECM interface with the medium channel, and $C_{\text {donor }}$ is the donor concertation of the dextran dye $(0.5 \mathrm{mg} / \mathrm{mL})$ (Fig. $2 \mathrm{c}$ ).

\section{Cisplatin Exposure}

To determine the toxic effect of cisplatin on RPTEC tubules in the OrganoPlate, medium of both channels (apical and basal) was replaced at day 6 after seeding with TOX medium (MEME alpha Modification (Sigma, M4526) supplemented with RPTEC Tox Supplement (Sigma, MTOXRTSUP), L-glutamine (1.87 mM, Sigma, G7513)) in the presence of $0,5,15,30,90,135$, or $270 \mu \mathrm{M}$ cisplatin (Sigma, P4394, stock: $5 \mathrm{mM}$ in $0.9 \% \mathrm{NaCl}$ (Sigma, S7653) in $\mathrm{H}_{2} 0$ ). After 48-h incubation on the rocker platform, phase contrast images were taken and the medium was sampled from the top channel. Samples from in- and outlet were pooled and used for the LDH activity assay. Next, tubes were incubated with WST-8 to determine cell viability. The barrier integrity of the exposed tubules was assessed consecutively of the WST-8 assay. After the exposures and viability measurements, the tubules were fixed with formaldehyde and stained with H2A.X, actin, and ZO-1.

\section{Lactate Dehydrogenase Activity Assay}

Lactate dehydrogenase (LDH) activity of the samples was determined using the Lactate Dehydrogenase Activity
Assay Kit (Sigma, MAK066) according to the manufacturer protocol. In short, the medium of the top in- and top outlet was pooled and $2 \mu \mathrm{L}$ was added in duplicate to a 384 well plate. In parallel, a concentration curve of the NADH standard was added. Next, $18 \mu \mathrm{L}$ LDH Assay Buffer was added to all sample wells to bring to an initial volume of $20 \mu$ l. After a short centrifugation of the plate, $20 \mu \mathrm{l}$ Master Reaction Mix were added to each well and mixed on a horizontal shaker in the plate reader. After $1 \mathrm{~min}$, the absorbance was measured at $450 \mathrm{~nm}$. While the plate was incubated, it was measured every 2 min until the value of the most active sample was higher than that of the highest standard (12.5 nmol/well). For the analysis, the LDH activity was determined using the following formula

LDH activity $=\frac{B \times \text { sample dilution factor }}{(\text { reaction time }) \times V}$

where $B$ is the amount (nanomole) of NADH generated between $t_{\text {initial }}$ and $t_{\text {final }}$, the reaction time is $t_{\text {final }}-t_{\text {initial }}$ (in minutes), and $V$ is the sample volume (in milliliters) added to the well.

\section{Cell Viability (WST-8 Assay)}

The cell viability of the cells was determined using the Cell Counting Kit-8 (Sigma, 96992). The WST-8 solution was diluted 1:11 with TOX medium and added to the channels of the OrganoPlate ( $30 \mu \mathrm{L}$ in- and outlets). After $18 \mathrm{~min}$ on the rocker platform and a 2-min flat incubation, the absorbance in the top in- and outlets was measured with the Multiskan ${ }^{\mathrm{TM}} \mathrm{FC}$ Microplate Photometer (Thermo scientific) at $450 \mathrm{~nm}$.

\section{Calcein-AM Efflux Inhibition}

Medium in all perfusion channels was replaced with $1 \mu \mathrm{M}$ calcein-AM (Life technologies, C3099, stock: $1 \mathrm{mM}$ in DMSO) in KHH buffer (Krebs-Henseleit (Sigma, K3753) + $10 \mathrm{mM}$ HEPES (Gibco, 15630) adjusted to $\mathrm{pH}$ 7.4) in the presence of $10 \mu \mathrm{M}$ cyclosporin A (Sigma, 30024, stock $5 \mathrm{mM}$ in DMSO), $500 \mu \mathrm{M}$ Digoxin (Fluka, 4599, stock $100 \mathrm{mM}$ in DMSO), or $0.5 \%$ DMSO (Sigma, D8418, vehicle control). After a 60-min incubation on the rocker platform, chips were washed one time with ice cold $\mathrm{KHH}$ buffer. In the next washing step, Hoechst 33342 (2 drop/mL, Life Technologies, R37605), $10 \mu \mathrm{M}$ PSC833 (Sigma, SML0572, stock: $5 \mathrm{mM}$ in DMSO), $10 \mu \mathrm{M}$ Ko143 (Sigma, K2144, stock $10 \mathrm{mM}$ in DMSO), and $10 \mu \mathrm{M}$ MK571 (Sigma, M7571, stock $10 \mathrm{mM}$ in $\mathrm{H}_{2} \mathrm{O}$ ) were added to the washing solution and the plate was imaged with the ImageXpress ${ }^{\circledR}$ Micro Confocal HighContent Imaging System.

\section{MRP2/4 Efflux Inhibition}

Medium in all perfusion channels was replaced with $1.25 \mu \mathrm{M}$ CMFDA (Molecular Probes, C7025, stock $2.5 \mathrm{mM}$ in DMSO) in the presence of 0, 10, 20, and $30 \mu \mathrm{M}$ MK571 (Sigma, M7571, stock $10 \mathrm{mM}$ in $\mathrm{H}_{2} \mathrm{O}$ ) in $\mathrm{KHH}$ buffer. After a 30-min incubation on the rocker platform, the chips were washed one time with ice cold KHH buffer. In the next washing step, Hoechst 33342 (2 drops/mL, 
a
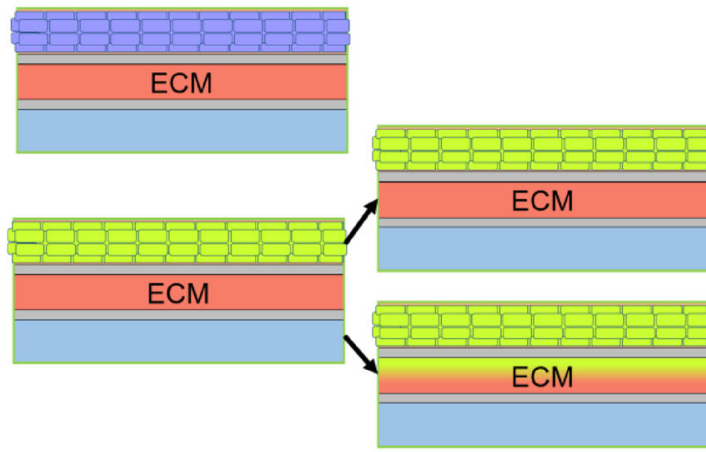

b

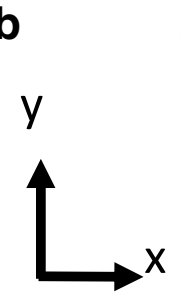

C
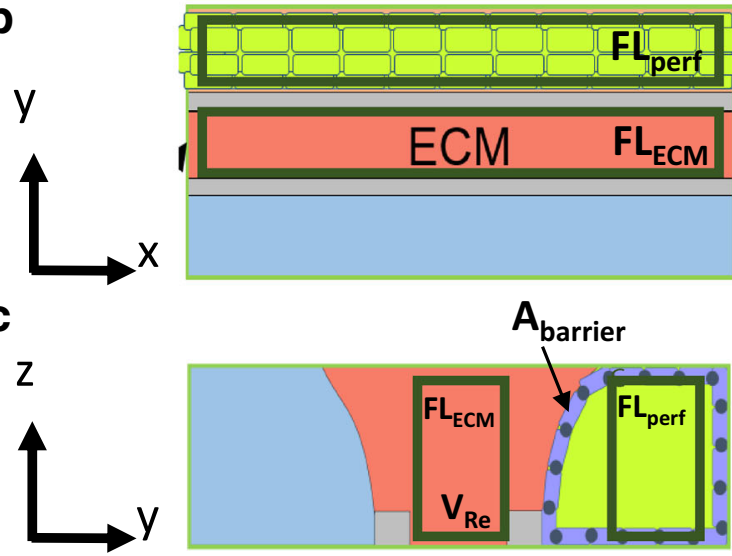

d
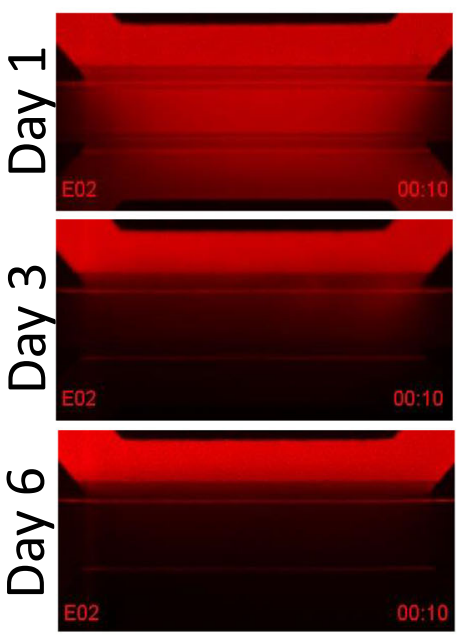

e

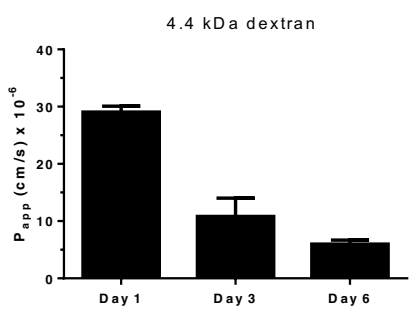

150 kDa dextran
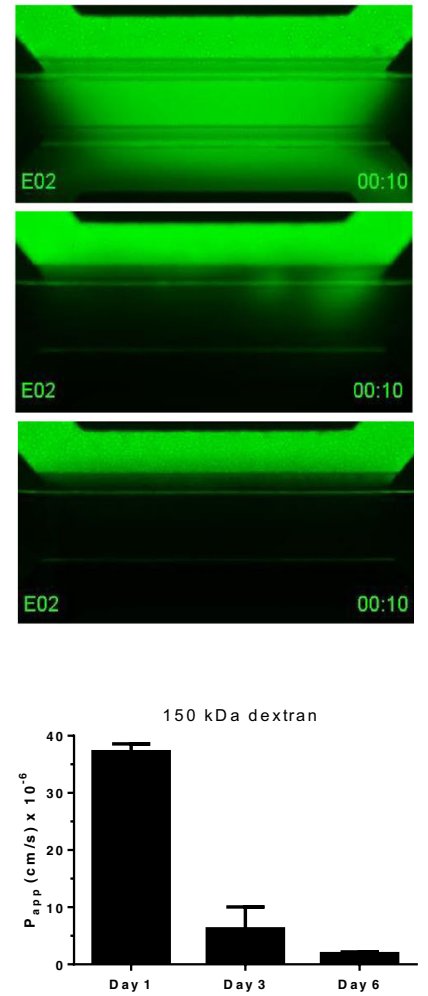

Fig. 2. Barrier integrity assay on tubular culture in the three-lane OrganoPlate platform. a The barrier integrity of the cell layer against the ECM was examined by perfusing the lumen of the tubule with dextran dyes. In healthy, leak-tight tubules, dyes remain in the lumen, whereas in leaky tubules, the dye enters the ECM channel. b The proportion of dye leaking through the tubule was monitored by measuring the fluorescence of the leaking dye into the gel. The gel channel intensity ( $\mathrm{FL}_{\mathrm{ECM}}$ ) was normalized for the intensity of dye in the perfusion channel $\left(\mathrm{FL}_{\text {perf }}\right) . \mathbf{c}$ Vertical illustration of the dye in the lumen of the tubule. $A_{\text {barrier }}$ is the area of the cell layer against the interface, $V_{\text {receiver }}\left(V_{\text {re }}\right)$ is the volume of the ECM behind the barrier which receives the dye. $\mathbf{d}$ Fluorescent images of the 10-min time point. Images were taken at day 1 , day 3 , and day 6 after seeding of RPTEC. At day 1, tubules did not form a barrier yet, whereas at day 3, the barrier is already partially formed. From day 6, the barrier is leak tight. e Apparent permeability $\left(P_{\text {app }}\right)$ of the RPTEC tubules at different days. For all measurements, the same chips were analyzed over time $(n=4)$. Error bars represent the standard deviation

Life Technologies, R37605), $10 \mu \mathrm{M}$ PSC833 (Sigma, SML0572, stock $5 \mathrm{mM}$ in DMSO), $10 \mu \mathrm{M}$ Ko143 (Sigma, K2144, stock $10 \mathrm{mM}$ in DMSO), and $10 \mu \mathrm{M}$ MK571 (Sigma, M7571) were added to the washing solution and the plate was imaged with the ImageXpress $®$ Micro Confocal High-Content Imaging System.

\section{6-NBDG Influx Inhibition}

Medium in the apical channel (Fig. 1) was replaced with OptiHBSS (1/3 Opti-MEM (Gibco, 11058-021), 2/3 HBSS (Sigma, H6648)) containing $500 \mu \mathrm{M}$ 6-(N-(7-Nitrobenz-2-oxa1,3-diazol-4-yl)amino)-6-Deoxyglucose (6-NBDG, Molecular Probes, N23106, lot 1704487, stock $10 \mathrm{mM}$ in $\mathrm{H}_{2} \mathrm{O}$ ) and 0, 20, 100 , or $500 \mu \mathrm{M}$ Phlorizin (Sigma, P3449, stock $200 \mathrm{mM}$ in Ethanol (JT Baker, 8025.2500PE)). All conditions contained $0.25 \%$ ethanol as vehicle. Medium in the basal channel was replaced with 6-NBDG-free medium, concentrations of phlorizin matched apical channel concentrations. After a 30min incubation on the rocker platform, cells were washed two times with ice cold OptiHBSS. In the second washing step, Hoechst 33342 (2 drops/mL, Life Technologies, R37605) was added to the washing solution and the plate was imaged with the ImageXpress ${ }^{\circledR}$ Micro Confocal High-Content Imaging System.

\section{Image Acquisition and Analysis of the Transport Experiments}

For the in-cell transport assays, plates were imaged with the ImageXpress ${ }^{\circledR}$ Micro Confocal High-Content Imaging System. A z-stack of $220 \mu \mathrm{m}$ with $10 \mu \mathrm{m}$ between each image plane was imaged with the FITC and the DAPI channel (Fig. S1). The intensity of the FITC signal of the cells growing against the ECM was analyzed with Fiji (13) and corrected for the background and cell number. Treated chips were normalized against vehicle control.

\section{Transepithelial Transport Assay}

Medium in the apical channel was replaced with medium containing $20 \mu \mathrm{M}$ cyclosporin A or $0.4 \%$ DMSO. Medium in the basal channel was replaced with TOX medium containing $10 \mu \mathrm{M}$ rhodamine 123 (Sigma, 83702, stock $50 \mathrm{mM}$ in ethanol) together with $20 \mu \mathrm{M}$ cyclosporin A or $0.4 \%$ DMSO. To determine the concentration of rhodamine 123, a 
concentration curve was added to unused chips. Tubules were incubated for $5 \mathrm{~h}$ on the rocker platform. After $3 \mathrm{~h}$ and after $5 \mathrm{~h}$, rhodamine 123 concentration was measured by imaging the top inlets with the FITC filter on the ImageXpress Micro XLS-C High Content Imaging Systems. The apparent permeability $\left(P_{\text {app }}\right)$ was calculated by using the formula

$$
P_{\text {app }}=\frac{\Delta C_{\text {receiver }} \times V_{\text {receiver }}}{\Delta \mathrm{t} \times \mathrm{A} \times C_{\text {donor }}}\left(\frac{\mathrm{cm}}{\mathrm{s}}\right) .
$$

$C_{\text {receiver }}$ is the measured intensity difference in the top wells between $t_{3 \mathrm{~h}}$ and $t_{5 \mathrm{~h}}, V_{\text {receiver }}$ is the receiving volume in the reservoirs of the top inlets, $t$ is the time difference $t_{5 \mathrm{~h}}-$ $t_{3 \mathrm{~h}}=2 \mathrm{~h}, A$ is the surface of the ECM interface with the medium channel, and $C_{\text {donor }}$ is the donor concertation of $10 \mu \mathrm{M}$ rhodamine 123 .

\section{Flow Simulation and Experimental Verification}

The platform described in this work uses a gravity-based perfusion system. The fluid flow rate and induced shear stress in the microfluidic channels of the OrganoPlate was estimated using a numerical model simulated in Python (Python Software Foundation, USA). This model calculates the induced pressure difference between two volumes of fluid, which are present in two microtiter plate wells that are connected by a microfluidic channel. The numerical model is described in more detail in the supplementary information. To validate the numerical model of the gravity-driven flow in the OrganoPlate, absorption was sequentially measured at $494 \mathrm{~nm}$ using a Fluorescein solution (Sigma, 46960, $10 \mu \mathrm{g} / \mathrm{mL}$ in water). For the verification, a 9603200B OrganoPlate (two-lane plate with $120 \times 200 \mu \mathrm{m}, w \times h$ channels) was used. The FITC solution was added to the channel system with $50 \mu \mathrm{L}$ in each in- and outlet. After tilting the plate at a set angle, the fluorescence of both wells was measured and compared with the associated simulated volumes.

\section{Statistics and Data Analysis}

Images were analyzed using Fiji (13). Data analysis was performed using Excel (Microsoft office 2016) and GraphPad Prism (GraphPad Software Inc., version 6.07). Error bars represent the standard deviation. Data were analyzed using one-way ANOVA followed by a Dunnett multiple comparison test which compares all treated chips to the control chips. Comparisons of two groups were done using the $t$ test. A $p$ value of $<0.05$ was considered to be significant. At least three technical replicates per data point were obtained.

\section{RESULTS}

\section{Development of a Proximal Tubule-on-a-Chip}

The platform we used to develop perfused 3D proximal tubules was the three-lane version of the OrganoPlate (Fig. 1a). The top part of this plate is a standard 384-well plate with a modified glass bottom. In the bottom of the OrganoPlate, 40 microfluidic chips are embedded. One chip consists of three 400$\mu \mathrm{m}$-wide and $220-\mu \mathrm{m}$-high channels separated by ridges, the phaseguides (14). First, an extracellular matrix (ECM) gel was loaded to the middle channel of the OrganoPlate. The liquefied
ECM entered the channels by capillary action and did not overflow to the adjacent channel through meniscus pinning on the phaseguide. The ECM is free-standing, allowing interrogation of the epithelial barrier function without interference of an artificial membrane. For the seeding procedure, a single cell suspension of RPTEC was added to one of the adjacent channels and cells could attach to the ECM by placing the plate on its side, in vertical position (Fig. 1a). After attachment of the cells, the plate was placed flat on a rocking platform. By positioning the in- and outlets of one chip on different heights, liquid flow was induced through the channels by leveling between the reservoirs (Fig. S5a). Fluid flows are bidirectional and pulsatile. Flow profiles have been simulated and experimentally verified (Fig. S5b). The change in fluorescence due to flow of FITC solution between wells showed a high correlation with the associated simulated volumes. Results show that mean flow rates of $2.02 \mu \mathrm{L} / \mathrm{min}$ could be achieved with a mean shear of 0.13 dyne $/ \mathrm{cm}^{2}$ (Fig. S5 c, d).

The seeding and culture conditions for proximal tubuleson-a-chip based on RPTEC cells were optimized by testing different settings for parameters, such as seeding density, ECM composition, medium composition, and perfusion height and angle. Figure $1 b$ shows optimal tube formation of RPTEC over time. As seen in Fig. S3, the flow is crucial for the tubule formation. Without any flow, tubule formation is not possible. In Fig. S4, the long-term viability of the RPTEC in the OrganoPlate is depicted. RPTEC are stable in the OrganoPlate up to day 11. After this time period, cells start invading the ECM which makes barrier-dependent assays impossible. Therefore, it was decided to perform all assays from day 6 to day 10 .

\section{Proximal Tubules-on-a-Chip Form Polarized Tight Barriers}

RPTEC tubules were cultured in the OrganoPlate for 710 days. As depicted in Fig. 1c, RPTEC formed a tubular structure with cells lining the ECM (curved part of the tubule) and the walls of the channel, thus having an open, perfused lumen. Confluent tubules formed tight junctions as visualized by the zona-occludence 1 tight junction (ZO-1) expression (15), and display primary cilia visualized by acetylated tubulin staining (16). A single cilium per cell was observed, located at the apical side of the cell layer pointing towards the lumen of the tubule (Fig. 1c). The tubules were also stained for Ezrin (17) that was expressed on the luminal side of the tubule (Fig. 1d). It is thus confirmed that the tubes are polarized in a correct manner, with the lumen being the apical side (corresponding to the preurine side in an in vivo situation) and the basal side being against the extracellular matrix (corresponding to the blood side in and in vivo situation).

Subsequently, the integrity of the epithelial barrier was investigated. To this end, a fluorescently labeled dextran was administered to the lumen of the tube. Leakage of the fluorescent dextran from the perfusion channel into the gel compartment was monitored and quantified in order to have a measure for the integrity of the epithelial cell monolayer (Fig. 2a-c). To monitor the tubule formation over several days, a higher molecular weight dextran (150 kDa FITC) and a lower molecular weight dextran (4.4 kDa TRITC) were used and leakage was assessed at day 1 and 3 and 6 days after seeding. At day 1, no barrier formation could be detected, whereas at day 3 the tubes were partly leak-tight. At day 6, 
the tubules were fully leak-tight for both dyes (Fig. 2d). After quantification of the signal at day 6 , the calculated $P_{\text {app }}$ (apparent permeability) of the tubules were $6 \times 10^{-6} \mathrm{~cm} / \mathrm{s}$ for the $4.4 \mathrm{kDa}$ dextran and $2 \times 10^{-6} \mathrm{~cm} / \mathrm{s}$ for the $150 \mathrm{kDa}$ dextran (Fig. 2e). A good barrier integrity of the tubule is crucial for assessing transport and directional toxicity as it allows interrogation and exposure of the apical and basolateral sides independently from one another.

\section{The Proximal Tubule-on-a-Chip Allows Nephrotoxicity Assessment}

Next, kidney tubules were assessed for toxicity response to cisplatin. Cisplatin is an anti-cancer drug, which is used for the treatment of tumors of the lung, ovary, testicles, and head and neck (18). The main route of the excretion of cisplatin is via the proximal tubules, which leads to a higher accumulation of the drug in the cells compared to other organs (19). Kidney tubules were exposed 6 days after seeding for $48 \mathrm{~h}$ to cisplatin at concentrations ranging from 5 to $270 \mu \mathrm{M}$. The toxic effect of cisplatin on the proximal tubules was determined through multiplexing several assays: phase contrast imaging, barrier integrity assessment, WST-8 viability measurement, LDH release and immunohistochemical staining of tight junctions, DNA damage, and cytoskeleton integrity. The morphology of the tubules was analyzed by phase contrast imaging and visually started to change at $270 \mu \mathrm{M}$ cisplatin concentration; at lower concentrations, no significant differences were observed (Fig. 3a). Cisplatin showed a dosedependent disruption of the barrier integrity of the tubes as determined by leakage of fluorescent dextran $4.4 \mathrm{kDa}$ and $150 \mathrm{kDa}$ (Fig. 3b, c) with a matching increase in calculated $P_{\text {app }}$ for both the small and large dextran (Fig. 3d).

In addition to measuring the impairment of the barrier integrity of the tubules, the cell viability was assessed using the live cell enzymatic activity WST-8 assay. A decrease in cell viability was observed at cisplatin concentrations of $30 \mu \mathrm{M}$ and higher, which was reduced to approximately $76 \%$ compared to the vehicle control at the highest concentration of $270 \mu \mathrm{M}$ (Fig. 3e). Consistent with this, a significant increase in $\mathrm{LDH}$ (lactate dehydrogenase) release into the lumen was detected at cisplatin concentrations of $30 \mu \mathrm{M}$ and higher (Fig. 3f). Immunohistochemical analysis of the exposed tubules further confirmed the toxicity of cisplatin. At concentrations of $30 \mu \mathrm{M}$ and higher, cisplatin caused increased DNA-damage (detected by H2A.X DNA-damage marker staining (20), Fig. 3g, h) and reduced expression of ZO-1 protein in the tight junctions (Fig. 3g, j). Changes in the actin cytoskeleton were observed from $5 \mu \mathrm{M}$ onwards (Fig. 3g, i).

\section{Active Substrate Transport Across the Epithelial Membrane}

The kidneys play a crucial role in eliminating drugs and metabolic waste products through excretion into the urine
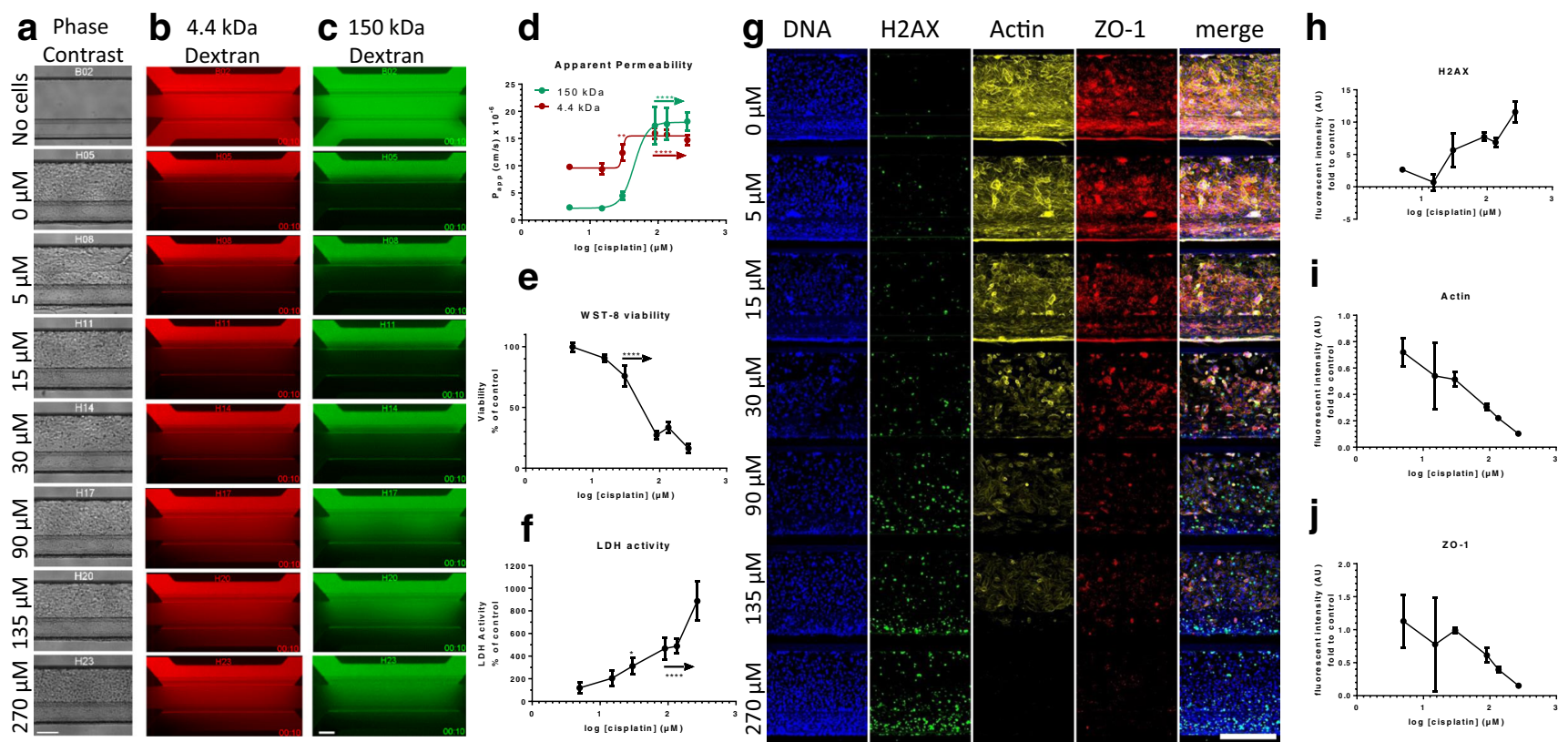

Fig. 3. Toxicant readouts after 48 -h cisplatin exposure. a Visual break down of the tube after $48 \mathrm{~h}$ of treatment at highest concentrations. Scale bar $=100 \mu \mathrm{m}$. b, c Perfusion of the lumen in the top channel with a lower sized dextran dye of $4.4 \mathrm{kDa}$ and a higher sized dextran dye of $150 \mathrm{kDa}$. From a concentration of $90 \mu \mathrm{M}$, the ECM behind the barrier is filled with dye, whereas at concentrations of $5 \mu \mathrm{M}$ and $15 \mu \mathrm{M}$, no difference compared to vehicle control can be seen. Scale bar $=200 \mu \mathrm{m}$. d For quantification of the barrier function, the apparent permeability $\left(P_{\text {app }}\right)$ was calculated. For both dextran sizes, a significant increase of the $P_{\text {app }}$ could be detected from $90 \mu \mathrm{M}(p<0.0001)$ compared to vehicle control. e To quantify viability of the cells, a WST- 8 assay was used. WST- 8 is reduced by the cells to an orange formazan product which can be measured with an absorbance reader at $450 \mathrm{~nm}$. Viability was significantly reduced $(p<0.0001)$ from $30 \mu \mathrm{M}$ compared to vehicle control. f LDH activity in the medium indicated the number of dead cells. The LDH assay detected, similar to the WST- 8 assay, a significant effect of cisplatin from $30 \mu \mathrm{M}(p<0.05)$. g Maximum projections or the immunostaining against H2A.X (DNA damage), actin (cytoskeleton), and ZO-1 (tight junction marker). Scale bar $=200 \mu \mathrm{m}$. $\mathbf{h}-\mathbf{j}$ Fluorescent intensity analysis of the summary projections of DNA damage marker H2A.X, actin cytoskeleton marker, and tight junction marker ZO-1. The intensity of all three markers is corrected for the background and nuclei count. Graphs show data of three chips per condition. Error bars represent the standard deviation 
(21). Many compounds require active transport by dedicated enzymes; the efficiency of which can be compromised by different drugs $(2,22)$.

To study the transporter functionality of kidney tubules in the OrganoPlate, calcein-AM and CMFDA were used to monitor P-gp and MRPs, respectively. These two transporters, which transport substrates into the apical lumen, are from the ATP-binding cassette family of transporters. Pgp mainly transports cationic as well as unconjugated xenobiotics whereas MRP is responsible for removing conjugated compounds from the body $(22,23)$. Calcein-AM is a substrate for $\mathrm{P}$-gp which is often used to assess the functionality of the transporter (23). Cell tracker reagent 5chloromethylfluorescein diacetate (CMFDA) is a compound for the MRP transporters (24). Both compounds enter the cells passively and are converted inside of the cells to the green fluorescent dyes calcein and GS-MF, respectively $(23,25)$. Cyclosporin A is an inhibitor of the P-gp transporter, whereas digoxin, which is a medication used to treat various heart conditions, is a substrate for P-gp (2) (Fig. 4a). MK571 is an inhibitor of the MRPs (2) (Fig. 4d).

To measure the influence of compounds on transport activity in the proximal tubule-on-a-chip, we set up fluorescent substrate-based activity assays (Fig. S1). For the analysis of the uptake of fluorescence inside of the cells lining the ECM, a z-stack of the tubule was imaged, and planes of the desired area are selected (Fig. S1e). After removal of the signal from out-of-focus cells, stacks were compressed, and the signal was integrated (Fig. S1f).
As both dyes enter the cells passively, RPTEC tubules were exposed to the dyes as well as their respective inhibitors from the apical as well as basal side. A $2.2 \pm 0.1$ - or $1.5 \pm 0.2$ fold increase in calcein accumulation was observed after coincubation with cyclosporin A or digoxin, respectively, confirming P-gp activity as both drugs competitively interact with the efflux pump (Fig. 4b, c). To monitor MRP-function, an efflux inhibitor cocktail of PSC833, MK571, and Ko143 was used to avoid redundancy of other transporters (23). A dose-dependent inhibition of GS-MF efflux was observed upon increasing MK571 concentrations, as shown in Fig. 4e, confirming MRP activity.

In addition to the efflux transporters, the glucose uptake by the sodium-glucose-linked transporter SGLT2 $(26,27)$ was evaluated using the fluorescent glucose analog 6-NBDG (28). SGLT2-mediated influx from tubular lumen into the cells was sensitive to inhibition by the SGLT inhibitor phlorizin (29) (Fig. 4f, g). This confirms the presence and activity of the SGLT2 transporter.

\section{The Proximal Tubule-on-a-Chip Allows Transepithelial Transport Assessment}

To further examine the capability of transepithelial transport across the epithelium of the 3D perfused proximal tubules, the flux of rhodamine123 from the basolateral to the apical compartment was assessed. Rhodamine123 is a substrate of P-gp, and its transport was analyzed in the absence and presence of the transport inhibitor cyclosporin A (30).
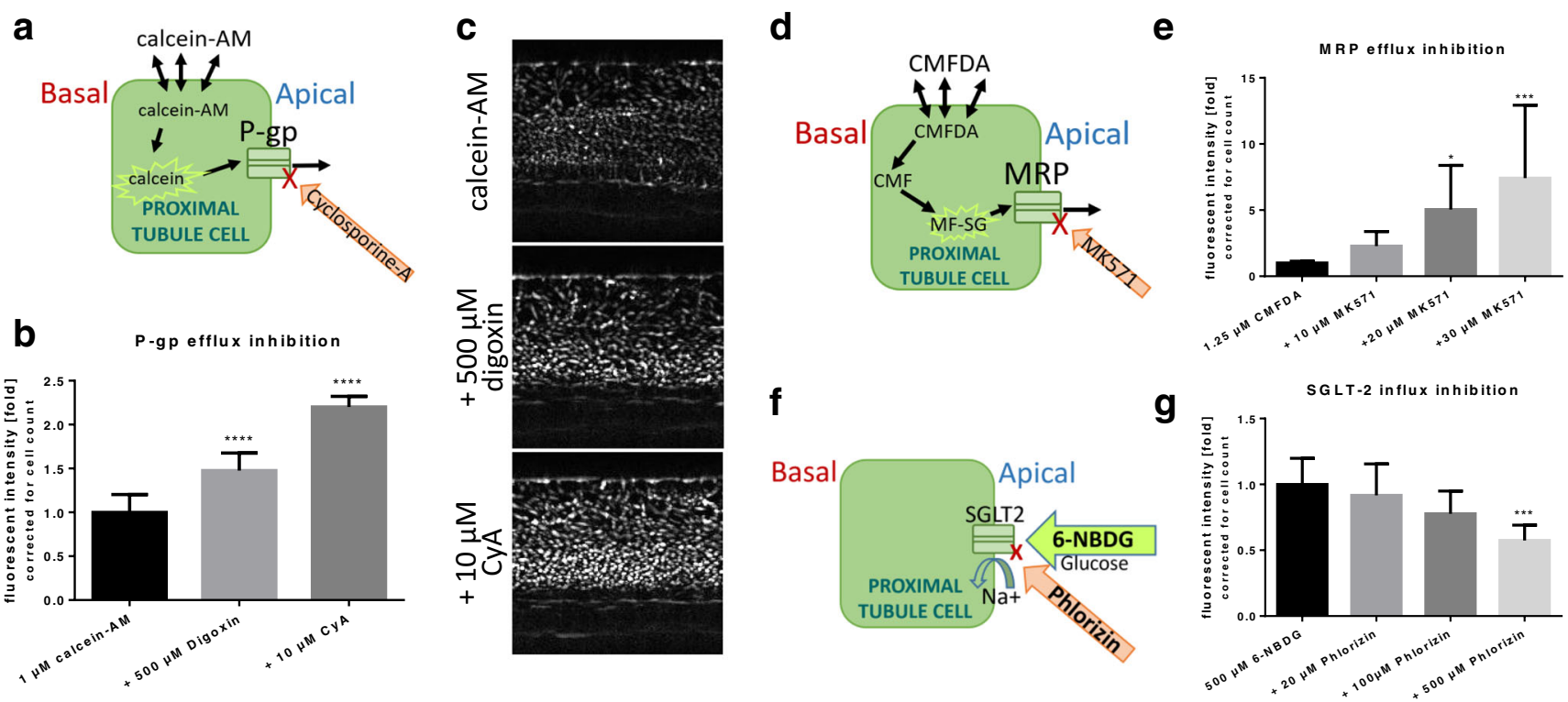

Fig. 4. Evaluation of P-gp, MRP, and SGLT2 transporter activity in proximal tubule-on-a-chip. a When the non-fluorescent calcein-AM enters the cell membrane, esterases in the cytoplasm cleave the acetoxymethyl (AM) ester group, which results in fluorescent calcein. Calcein-AM is pumped out of the cell by the P-glycoprotein-transporter (P-gp). b Inhibition of calcein-AM efflux: RPTEC were incubated for $1 \mathrm{~h}$ with $1 \mu \mathrm{M}$ calcein-AM \pm inhibitors. Ten micromolar of cyclosporin A showed the highest inhibitory effect followed by $500 \mu \mathrm{M}$ digoxin. $\mathbf{c}$ Z-Projections of representative images of the calcein-AM exposed RPTEC area. A higher fluorescent signal could be observed in the presence of transport inhibitors. Scale bar $=200 \mu \mathrm{m}$. d Similar to calcein-AM, non-fluorescent CMFDA enters the cells passively. Inside the cells, CMFDA is transformed to fluorescent MRP substrate GS-MF. e Dose-dependent efflux inhibition of GS-MF by MK571 resulted in a significant increase of fluorescent signal inside the cells. $f$ Influx of fluorescent glucose analog 6-NBDG is inhibited by phlorizin. 6-NBDG influx is mediated by the sodium-dependent SGLT2 transporter. g 6-NBDG influx into RPTEC was significantly inhibited by $500 \mu \mathrm{M}$ phlorizin, and a dose-dependent trend was observed when inhibited with 20 and $100 \mu \mathrm{M}$ phlorizin. $* * p<0.01 * * * p<0.001$ and $* * * * p<0.0001$ one-way ANOVA with Dunnett's comparison test. Each of the three graphs shows combined data of two independent experiments with two to five chips per condition. Error bars represent the standard deviation 
The experimental set-up is shown in Fig. 5a, b. Rhodamine123 was administered to the basal perfusion channel and measured in the perfusion medium on the apical side. In order to have a highest signal-to-noise ratio, the fluorescent signal was measured in apical-side in- and outlet, where the liquid column was largest. Inhibition of transport by cyclosporine A reduced fluorescence signal in the apical-side inand outlets as shown by representative images in Fig. 5c. It is known that cyclosporin A has a toxic effect on the kidney. Therefore, its effect on the barrier integrity was assessed. It was found that a concentration of $20 \mu \mathrm{M}$ cyclosporin A was determined as safe as transport inhibitor, supported by the absence of an effect on barrier integrity (Fig. S2). The $P_{\text {app }}$ of rhodamine123 changed from $3.4 \times 10^{-5} \pm 0.1 \times 10^{-5} \mathrm{~cm} / \mathrm{s}$ without inhibitor to $2.2 \times 10^{-5} \pm 0.1 \times 10^{-5} \mathrm{~cm} / \mathrm{s}$ with cyclosporin A, indicating (inhibition of) active transport (Fig. 5d). This is a clear demonstration that transepithelial transport can be assessed in the system.

\section{DISCUSSION AND OUTLOOK}

We reported the development of a functional proximal tubule-on-a-chip model which can be implemented for routine assessment of kidney toxicity and drug-drug interaction studies. The OrganoPlate platform allows parallel culture and assessment of 40 independent kidney tubules. This is important in toxicological studies or in compound testing as it allows for proper controls, replica's, and dilutions series. Induction of flow is realized by passive leveling on an interval rocker system, which is an easy to use alternative to complex pump solutions. Other than the rocking platform, no further specialized lab equipment is needed. As the format of the platform is a standard microtiter layout of a 384-well plate, it is fully compatible with most readers, microscopes, and robot handling equipment. The platform is fully pipet operated and media changes as well as reagent additions are non-invasive. Physiologically relevance of the system is enhanced by culturing epithelial cells directly against an extracellular matrix (ECM) mimic in a manner that is free of artificial membranes, under application of perfusion flow and by mimicking the 3D morphology.

A critical aspect in the concept is the selective patterning of extracellular matrix in the chips. In order to do this, surface tension techniques have been employed that allow selective ECM priming using pipetting means only. As a consequence thereof, only part of the tube is exposed to the ECM (curved part). This does not hamper the measurement results as the barrier and the rhodamine transport assay exclusively consider the cell layer against the extracellular matrix. Other systems are known from literature that have full ECM embedment (31); however, such systems come at the cost of throughput and ease of handling, as it is impossible to create a lumen in an ECM gel with pipetting steps only.

Similar choices were made with respect to the dimensions of the proximal tubule. In this study, the diameter of the tubules is $400 \mu \mathrm{m}$, which is significantly larger than the in vivo proximal tubules (approximately $60 \mu \mathrm{m}$ (32)). However, the choice for larger dimensions allowed a greater sensitivity of assays, particularly those that are executed off-chip.

We showed the multiplexing assays in a microfluidic format. The barrier integrity could be monitored in real time by adding a reporter dye. In parallel, cellular enzymatic activity (WST-8) and LDH release could be measured. All three assays yielded a similar result to cisplatin exposure, indicating the robustness of the model. Immunohistochemical staining was also used in a multiplex manner for assessment a

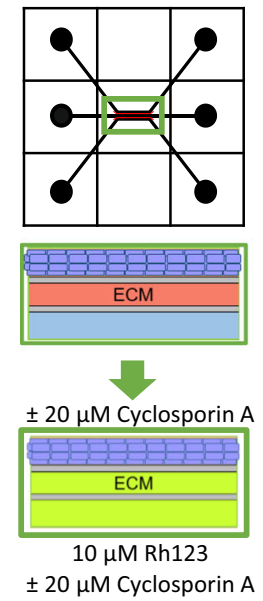

b
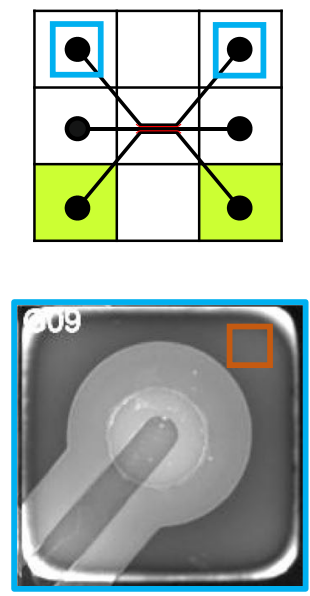

C

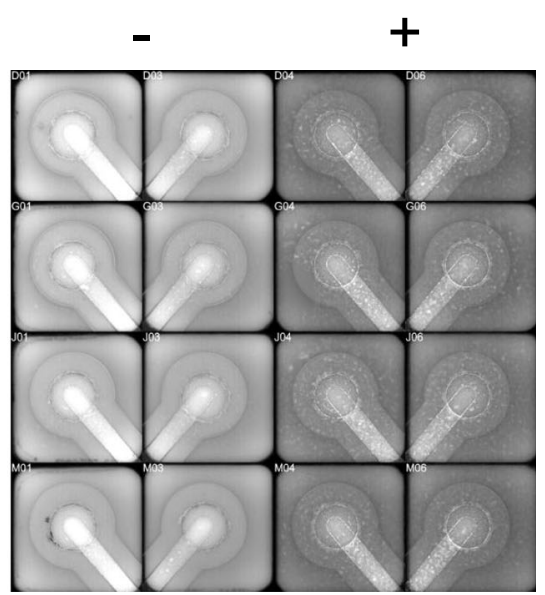

d

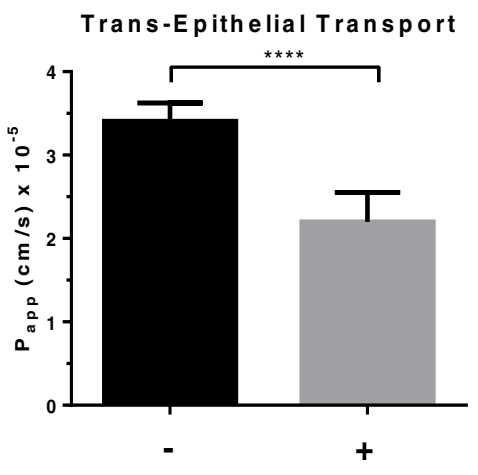

Fig. 5. Transepithelial transport of rhodamine 123. a Ten micromolar of rhodamine 123 was added solely to the basal side of the tubule with or without $20 \mu \mathrm{M}$ cyclosporin A present at both sides of the tube. b After 3 and $5 \mathrm{~h}$, images were taken from the inlets guiding to the lumen of the tubule (blue squares: measured wells). The intensity above the glass bottom of the wells was measured (orange square: area selection). To determine the concentration of rhodamine 123 in the top wells, spiked samples were added to empty chips on the same plate and analyzed. The resulting concentrations were used to calculate the $P_{\text {app }}$ of the transport of rhodamine 123. c Images of the top in- and outlets or the OrganoPlate show the apical fluorescent signals after 5-h incubation of rhodamine 123 on the basal side of the tubule. A decreased fluorescent signal was observed after co-incubation with cyclosporin A, as shown in the top inlets. -: without transport inhibition, +: with $20 \mu \mathrm{M}$ cyclosporin A addition (apical and basal). d A significant decrease in $P_{\text {app }}$ was observed when adding rhodamine 123 together with $20 \mu \mathrm{M}$ cyclosporin A. $* * * * p<0.0001$. Data are combined from two independent experiments with five to nine chips per plate and per condition. Error bars represent the standard deviation 
of the effect of cisplatin on the tight junctions (ZO-1), cytoskeleton (actin), and DNA damage (H2A.X). Although some showed higher sensitivity than the live assays, the stains represent an end-point assay and require much more effort to execute properly. Moreover, only a limited number of stains could be multiplexed in the same model, requiring replica's to be used for different analyses.

The multiplexed live assays used for acute kidney toxicity detection here are compatible with chronic toxicity assessments. For example, the barrier integrity assays were previously used to study 5-day exposure of gut tubules to toxicants (33). Noninvasive assays are important to study chronic effects of drugs that only exert negative effects after prolonged use. The proximal tubule model developed here together with multiplexed assays and the possibility for repeated dosing of the model allows for future longitudinal studies.

In this publication, we used the microfluidic system for growing tubular structures. In addition to that, other cell types can be embedded into the ECM. An example of functional 3D networks of neurons and glia embedded in the ECM of one channel with an adjacent medium channel is shown by Wevers et al. (34), and Lanz et al. studied the behavior of breast cancer cells grown in an ECM (35). The method of culturing cells embedded in the ECM can be easily combined with the model presented here: instead of culturing the RPTEC against a pure ECM, supporting cells can be added into the ECM. In addition to this, the complexity of the model could be further increased by growing an endothelial tubule in the basal-side perfusion channel. Ultimately, we aim to combine endothelium, fibroblasts, and podocytes to have a fully functional kidney-on-a-chip model.

We showed two different possibilities to monitor the transport of the proximal tubules. First, we measured and analyzed the signal of compounds retained by the cells lining the ECM layer. Second, we showed feasibility of transepithelial transport studies on the RPTEC containing tubules using the fluorescent substrate, rhodamine123. Both types of experiments show clear transporter functionality, whereas the latter is the most complex functional assay. We have not assessed organic anion transporter (OAT) expression by the RPTEC used in this study, but $2 \mathrm{D}$ evaluation by Suter-Dick et al. (36) showed no response to tenofovir, indicating absence of the OAT1 transporter. This limits the use of the cell line for assessment of transport of organic ions. To compensate for this, we also implemented a cell line overexpressing the organic anion transporter 1 (ciPTEC, (Vriend et al. 37)). In future work, we will compare the performance of both cell models in response to a range of blinded compounds.

For future studies, transport capabilities will be shown also for non-fluorescent substrates. To achieve this goal, radiolabeled compounds or mass spectrometry can be used to analyze and quantify transport. In this manner, a much wider range of compounds and transporter functionality can be investigated. These off-plate assays will require sampling of the perfusate instead of the microscope-based readouts used here. The use of microfluidic chips requires limited amounts of cells and medium, which is positive in the light of use of valuable materials with restricted access. However, for certain analysis methods, the small sample volumes could be limiting. Detection of transported compounds will depend on the sensitivity of the analysis method (e.g., mass spectrometry). Other off-plate analyses as for example qRT-PCR are feasible through pooling of chip lysates. Such functional assays will be of great support for in vitro to in vivo extrapolation.

In summary, we developed a user-friendly, functional kidney-on-a-chip model that can be used to study the effect of compounds in 40 parallel cultured renal tubules. The tubules could be assessed for barrier function by fluorescent imaging and multiplexed with a range of assays including viability, $\mathrm{LDH}$ leakage, and immunohistochemical staining. In addition, transporter activity was shown by means of transport inhibition studies for both substrate uptake, as well as transcellular transport. The functionality of the platform in combination with the ease of handling and decent throughput makes this a useful platform for studying nephrotoxicity, compound excretion, drug-drug interaction studies, and disease mechanisms.

\section{ACKNOWLEDGEMENTS}

This project was financially supported by NC3Rs (National Center for the Replacement, Refinement and Reduction of Animals in Research), UK, under the Crack-it challenge 15 (Nephrotube, project no. 37497-25920). We thank Professor Dr. Hans Tanke for the use of the imaging facilities at the Department of Molecular Cell Biology, Leiden University Medical Center, and Joop Wiegant and Annelies van der Laan for their assistance.

\section{COMPLIANCE WITH ETHICAL STANDARDS}

Conflict of Interest This publication contains original work. The authors M.K. Vormann, L. Gijzen, S. Hutter, L. Boot, A. Nicolas, A. vd Heuvel, C. Ng, B. de Wagenaar, S.J. Trietsch, J. Joore, P. Vulto, and H.L. Lanz are or were employees of Mimetas B.V. This

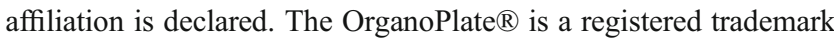
of MIMETAS BV.

Open Access This article is distributed under the terms of the Creative Commons Attribution 4.0 International License (http://creativecommons.org/licenses/by/4.0/), which permits unrestricted use, distribution, and reproduction in any medium, provided you give appropriate credit to the original author(s) and the source, provide a link to the Creative Commons license, and indicate if changes were made.

\section{REFERENCES}

1. Lauralee Sherwood. Human physiology: from cells to systems [internet]. Brooks Cole; 1997. 880 p. Available from: https:// www.amazon.com/Human-Physiology-Systems-Lauralee-Sherwood/dp/0314092455.

2. Morrissey KM, Stocker SL, Wittwer MB, Xu L, Giacomini KM. Renal transporters in drug development. Annu Rev Pharmacol Toxicol. 2013;53(1):503-29. [cited 2018 Jan 29]; Available from: http://www.ncbi.nlm.nih.gov/pubmed/23140242

3. Wilmer MJ, Ng CP, Lanz HL, Vulto P, Suter-Dick L, Masereeuw R. Kidney-on-a-chip technology for drug-induced 
nephrotoxicity screening. Trends Biotechnol. 2015;xx:1-15. Available from:. https://doi.org/10.1016/j.tibtech.2015.11.001.

4. Homan KA, Kolesky DB, Skylar-Scott MA, Herrmann J, Obuobi H, Moisan A, et al. Bioprinting of 3D convoluted renal proximal tubules on perfusable chips. Sci Rep. 2016;6:34845. Available from:. https://doi.org/10.1038/srep34845.

5. Vedula EM, Alonso JL, Arnaout MA, Charest JL. A microfluidic renal proximal tubule with active reabsorptive function. PLoS One. 2017;12(10):1-15.

6. Jang K-J, Mehr AP, Hamilton GA, McPartlin LA, Chung S, Suh K-Y, et al. Human kidney proximal tubule-on-a-chip for drug transport and nephrotoxicity assessment. Integr Biol (Camb). 2013;5(9):1119-29. Available from: http://www.ncbi.nlm.nih.gov/ pubmed/23644926

7. Qu Y, An F, Luo Y, Lu Y, Liu T, Zhao W, et al. A nephron model for study of drug-induced acute kidney injury and assessment of drug-induced nephrotoxicity. Biomaterials [Internet]. 2017; Available from: http://linkinghub.elsevier.com/retrieve/pii/S014296121730741X.

8. van Meer BJ, de Vries H, Firth KSA, van Weerd J, Tertoolen LGJ, Karperien HBJ, et al. Small molecule absorption by PDMS in the context of drug response bioassays. Biochem Biophys Res Commun. 2017;482(2):323-8. Available from. https://doi.org/10.1016/j.bbrc.2016.11.062.

9. van Duinen V, Trietsch SJ, Joore J, Vulto P, Hankemeier T. Microfluidic 3D cell culture: from tools to tissue models. Curr Opin Biotechnol. 2015;35:118-26. Available from: http:// www.ncbi.nlm.nih.gov/pubmed/26094109

10. Junaid A, Mashaghi A, Hankemeier T, Vulto P. An end-user perspective on Organ-on-a-Chip: assays and usability aspects. Curr Opin Biomed Eng. 2017;1:15-22. Available from: http:// linkinghub.elsevier.com/retrieve/pii/S246845111730003X

11. Trietsch SJ, Israëls GD, Joore J, Hankemeier T, Vulto P. Microfluidic titer plate for stratified 3D cell culture. Lab Chip. 2013;13(18):3548-54. [cited 2016 Apr 5]. Available from: http:// www.ncbi.nlm.nih.gov/pubmed/23887749

12. Li S, Zhao J, Huang R, Steiner T, Bourner M, Mitchell M, et al. Development and application of human renal proximal tubule epithelial cells for assessment of compound toxicity. Curr Chem Genomics Transl Med. 2017;11:19-30. Available from: http:// www.ncbi.nlm.nih.gov/pubmed/12234291

13. Schindelin J, Arganda-Carreras I, Frise E, Kaynig V, Longair M, Pietzsch T, et al. Fiji: an open-source platform for biologicalimage analysis. Nat Methods. 2012;9(7):676-82. [cited 2014 Jul 9]. Available from: http://www.nature.com/doifinder/ 10.1038/nmeth.2019

14. Vulto P, Podszun S, Meyer P, Hermann C, Manz A, Urban GA. Phaseguides: a paradigm shift in microfluidic priming and emptying. Lab Chip. 2011;11(9):1596-602. [cited 2016 Apr 5]. Available from: http://www.ncbi.nlm.nih.gov/pubmed/21394334

15. Stevenson BR, Siliciano JD, Mooseker MS, Goodenough DA. Identification of Z0-1: a high molecular weight polypeptide associated with tight junction (zonula occludens) in a. J Cell Biol. 1986;103(September):755-66.

16. Raghavan V, Weisz OA. Flow stimulated endocytosis in the proximal tubule. Curr Opin Nephrol Hypertens. 2015;24(4):35965. Available from: http://www.ncbi.nlm.nih.gov/pubmed/ $26050123 \% 5 \mathrm{Cn}$. http://www.pubmedcentral.nih.gov/ articlerender.fcgi? artid=PMC4494861

17. Berryman M, Franck Z, Bretscher A. Ezrin is concentrated in the apical microvilli of a wide variety of epithelial cells whereas moesin is found primarily in endothelial cells. J Cell Sci. 1993;105(4):1025-43.

18. Giaccone G. Clinical perspectives on platinum resistance. Drugs. 2000;59(4):9-17. Available from:. https://doi.org/ 10.2165/00003495-200059004-00002.

19. Oh GS, Kim HJ, Shen AH, Lee SB, Khadka D, Pandit A, et al. Cisplatin-induced kidney dysfunction and perspectives on improving treatment strategies. Electrolyte Blood Press. 2014;12(2):55-65.
20. Kuo LJ, Yang L-X. Gamma-H2AX - a novel biomarker for DNA double-strand breaks. In Vivo. 2008;22(3):305-9.

21. Masereeuw R, Russel FGM. Regulatory pathways for ATPbinding cassette transport proteins in kidney proximal tubules. AAPS J. 2012;14(4):883-94.

22. Giacomini KM, Tweedie D. Membrane transporters in drug development. Nat Rev Drug Discov. 2010;9(March):215-36.

23. Caetano-Pinto P, Janssen MJ, Gijzen L, Verscheijden L, Wilmer MJGG, Masereeuw R. Fluorescence-based transport assays revisited in a human renal proximal tubule cell line. Mol Pharm. 2016;13(3):933-44. Available from: http://pubs.acs.org/doi/abs/ 10.1021/acs.molpharmaceut.5b00821

24. Jenkinson SE, Chung GW, van Loon E, Bakar NS, Dalzell AM, Brown CDA. The limitations of renal epithelial cell line HK-2 as a model of drug transporter expression and function in the proximal tubule. Pflugers Arch - Eur J Physiol 2012;464(6):601-11.

25. Lindenmaier $\mathrm{H}$, Becker $\mathrm{M}$, Haefeli WJ. Interaction of progestins with the human multidrug resistance-associated protein 2 (MRP2). Drug Metab. 2005;33(11):1576-9. Available from: http://dmd.aspetjournals.org/content/33/11/1576.full

26. Wood IS, Trayhurn P. Glucose transporters (GLUT and SGLT): expanded families of sugar transport proteins. $\mathrm{Br} \mathbf{J}$ Nutr. 2003;89(01):3. Available from: http://www.journals.cambridge.org/ abstract_S0007114503000023

27. Vallon V, Platt KA, Cunard R, Schroth J, Whaley J, Thomson SC, et al. SGLT2 mediates glucose reabsorption in the early proximal tubule. J Am Soc Nephrol. 2011;22(1):104-12. Available from: http://www.jasn.org/cgi/doi/10.1681/ASN.2010030246

28. Jung D-W, Ha H-H, Zheng X, Chang Y-T, Williams DR. Novel use of fluorescent glucose analogues to identify a new class of triazine-based insulin mimetics possessing useful secondary effects. Mol BioSyst. 2011;7(2):346-58.

29. Ehrenkranz JRL, Lewis NG, Kahn CR, Roth J. Phlorizin: a review. Diabetes Metab Res Rev. 2005;21(1):31-8.

30. Sharom FJ. The P-glycoprotein multidrug transporter. Gen Pharmacol. 2011;27(8):1283-91.

31. Weber EJ, Chapron A, Chapron BD, Voellinger JL, Lidberg KA, Yeung CK, et al. Development of a microphysiological model of human kidney proximal tubule function. Kidney Int. 2016;90(3):627-37. Available from:. https://doi.org/10.1016/ j.kint.2016.06.011.

32. Liebich H-G. Funktionelle Histologie der Haussäugetiere und Vögel: Lehrbuch und Farbatlas für Studium und Praxis [internet]. 5th ed. Schattauer: Stuttgard; 2010. Available from: https://books.google.nl/ books?id=ATo7t_sGC7IC\&dq=durchmesser+proximaler+tubulus\&source $=$ gbs_navlinks_S

33. Trietsch SJ, Naumovska E, Kurek D, Setyawati MC, Vormann MK, Wilschut KJ, et al. Membrane-free culture and real-time barrier integrity assessment of perfused intestinal epithelium tubes. Nat Commun. 2017;8(1):262. Available from: http:// www.nature.com/articles/s41467-017-00259-3

34. Wevers N, Trietsch SJ, Vught RV, Joore J, Vulto P, Lanz H, et al. 3D networks of iPSC-derived neurons and glia for highthroughput neurotoxicity screening. Toxicol Lett. 2016;258:S157.

35. Lanz HL, Saleh A, Kramer B, Cairns J, Ng CP, Yu J, et al. Therapy response testing of breast cancer in a $3 \mathrm{D}$ high-throughput perfused microfluidic platform. BMC Cancer. 2017;17(1):709. Available from: http://bmccancer.biomedcentral.com/articles/10.1186/ s12885-017-3709-3

36. Suter-Dick L, Mauch L, Ramp D, Caj M, Vormann MK, Hutter S, et al. Combining extracellular miRNA determination with microfluidic 3D cell cultures for the assessment of nephrotoxicity: a proof of concept study. AAPS J. 2018;20:86. https://doi.org/ 10.1208/s12248-018-0245-2.

37. Vriend J, Nieskens TTG, Vormann MK, van den Berge BT, van den Heuvel A, Russel FGM, et al. Screening of drug-transporter interactions in a $3 \mathrm{D}$ microfluidic renal proximal tubule on a chip. AAPS J. 2018;20:87. https://doi.org/10.1208/s12248-018$0247-0$. 